CERN-PPE/94-75

20 May 1994

\title{
KAON INTERFEROMETRY IN HEAVY-ION COLLISIONS AT THE CERN SPS
}

\author{
H. Beker ${ }^{1, a)}$, H. Bøggild ${ }^{2)}$, J. Boissevain ${ }^{3)}$, M. Cherney ${ }^{4)}$, J. Dodd ${ }^{5)}$, S. Esumi ${ }^{6}$, \\ C.W. Fabjan ${ }^{1)}$, D.E. Fields ${ }^{3)}$, A. Franz ${ }^{1)}$, K.H. Hansen ${ }^{2)}$, B. Holzer ${ }^{1)}$, T. Humanic ${ }^{7)}$, \\ B. Jacak ${ }^{3)}$, R. Jayanti ${ }^{8, b)}$, H. Kalechofsky ${ }^{8, b)}$, T. Kobayashi ${ }^{9, c)}$, R. Kvatadze ${ }^{1, d)}$, \\ Y.Y. Lee ${ }^{8, b)}$, M. Leltchouk ${ }^{5)}$, B. Lörstad ${ }^{10)}$, N. Maeda $^{6)}$, A. Medvedev ${ }^{5)}$, Y. Miake ${ }^{11, e)}$, \\ A. Miyabayashi ${ }^{10)}$, M. Murray ${ }^{12)}$, S. Nagamiya ${ }^{5)}$, S. Nishimura ${ }^{6)}$, E. Noteboom ${ }^{4)}$, \\ S.U. Pandey ${ }^{8, b)}$, F. Piuz ${ }^{1)}$, V. Polychronakos ${ }^{11)}$, M. Potekhin ${ }^{5)}$, G. Poulard ${ }^{1)}$, \\ A. Sakaguchi ${ }^{6)}$, M. Sarabura ${ }^{3)}$, K. Shigaki ${ }^{9, f)}$, J. Simon-Gillo ${ }^{3)}$, W. Sondheim ${ }^{3)}$, \\ T. Sugitate ${ }^{6)}$, J.P. Sullivan ${ }^{3)}$, Y. Sumi ${ }^{6)}$, H. Sletten ${ }^{1)}$, H. van Hecke ${ }^{3)}$, W.J. Willis ${ }^{5)}$ and \\ K. Wolf ${ }^{12)}$

\begin{abstract}
$K^{+} K^{+}$and $K^{-} K^{-}$correlations from $\mathrm{S}+\mathrm{Pb}$ collisions at $200 \mathrm{GeV} / c$ per nucleon and $K^{+} K^{+}$correlations from $\mathrm{p}+\mathrm{Pb}$ collisions at $450 \mathrm{GeV} / c$ per nucleon, are presented as measured by the focusing spectrometer of the NA44 experiment at CERN. Multidimensional fits are performed in order to characterize the kaon-emission volume, which is found to be smaller than the pion-emission volume.
\end{abstract}

(Submitted to Z. Phys.)

\footnotetext{
1) CERN, CH-1211 Geneva 23, Switzerland.

2) Niels Bohr Institute, DK-2100 Copenhagen, Denmark.

3) Los Alamos National Laboratory, Los Alamos, NM 87545, USA.

4) Creighton University, Omaha, NE, USA.

5) Columbia University, New York, NY 10027, USA.

6) Hiroshima University, Higashi-Hiroshima 724, Japan.

7) Ohio State University, Columbus, OH 43210, USA.

8) University of Pittsburgh, Pittsburgh, PA 15260, USA.

9) National Laboratory for High Energy Physics, Tsukuba 305, Japan.

10) University of Lund, S-22362 Lund, Sweden.

11) Brookhaven National Laboratory, Upton, NY 11973, USA.

12) Texas A\&M University, College Station, TX 77843, USA.

a) Present address: Rome I Institute, Rome I-00185, Italy

b) Also at Ohio State University, Columbus, OH 43212, USA.

c) Now at Riken Linac Laboratory, Riken, Saitama 351-01, Japan.

d) Visitor from Tbilisi State University, Tbilisi, Rep. of Georgia.

e) Now at Tsukuba University, Tsukuba 305, Japan.

f) University of Tokyo, Tokyo 113, Japan.
} 
Two-particle intensity interferometry provides a method for measuring the spacetime extent of a particle-emitting source when the emitted radiation is at least partially incoherent [1-3]. Intensity interferometry measures the freeze-out volume when the particles decouple from the source. This effect is well established for pions. Charged kaons are less affected by resonance decay than pions and could provide a cleaner signal of the source [4, 5]. Furthermore, the different total cross-sections of $K^{+}, K^{-}$, and $\pi^{+}$with nuclear matter may allow the investigation of the source at different decoupling times [6].

Experiment NA44 is optimized for the study of identified single- and two-particle distributions at mid-rapidity. Excellent particle identification limits contamination to the $1 \%$ level. Since it is a focusing spectrometer, the acceptance for pairs of particles with small momentum difference is optimized, allowing small statistical uncertainties in the two-particle correlation function in the region of the signal from Bose-Einstein correlations. Our statistics permit multi-dimensional fits, which are more sensitive to interesting dynamics, less influenced by relativistic effects, and more plausibly related to emission volumes than correlations analysed in the four-momentum difference $\left(Q_{\text {inv }}\right)$ of the two particles which represents an average over all directions of the pairs within the acceptance of the experiment [7, 8]. A Gaussian parametrization is frequently applied to fit two-particle correlation functions [3]; however an exponential distribution could arise owing to particle-production dynamics, such as string-breaking [9] or decay of resonances [10-12]. The RQMD event generator [13], which simulates heavy-ion collisions, predicts that fewer kaons are produced from long-lived resonances than pions [14].

\section{EXPERIMENTAL SET-UP}

The layout of the experiment is shown in Fig. 1. The focusing spectrometer of the NA44 experiment has been described in detail elsewhere [15]. Two dipole magnets (D1 and D2) and three quadrupoles (Q1, Q2, and Q3) create a magnified image of the target in the spectrometer. Only one charge sign can be detected in the spectrometer at a time. The momentum range selected in this analysis covers a band of $\pm 20 \%$ at its nominal momentum setting of $6 \mathrm{GeV} / c$.

The beam rate and time-of-flight start signal for a sulfur beam are determined using a Cherenkov beam counter labelled CX in Fig. 1. The intrinsic time resolution of the Cherenkov beam counter is approximately 35 ps [16]. For the proton beam, the time-offlight start signal is determined using a forward scintillator counter, $T_{0}$. For the sulfur beam $\mathrm{T}_{0}$ serves as a trigger device, to signal 'minimum bias' events (at least one minimum ionizing particle) or 'central' events, for which the particle multiplicity as measured by the total pulse height in $\mathrm{T}_{0}$ is large. A silicon pad detector is used to measure the charged-particle multiplicity distribution with $2 \pi$ azimuthal acceptance in the pseudorapidity range $1.5<\eta<3.3$.

The spectrometer uses three highly segmented scintillator hodoscopes, labelled H1, H2, and H3 [17] in Fig. 1, for tracking and time-of-flight measurements. In this analysis we use only the time measured on $\mathrm{H} 3$ for particle identification with a time resolution of approximately $100 \mathrm{ps}$. The Cherenkov detectors are used to provide a kaon trigger by vetoing events containing electrons, muons, or pions in the spectrometer acceptance. Muons from kaon decays after the Cherenkov counters are vetoed if they are above the Cherenkov momentum threshold $1.9 \mathrm{GeV} / \mathrm{c}$ or if they do not form straight line trajectories.

Four wire chambers are used for momentum calibration in conjunction with the third dipole magnet. A uranium-scintillator calorimeter (UCAL), partitioned into elec- 
tromagnetic and hadronic sections, allows identification of $e, \pi$, and $\mu$, and is used for rejecting electrons in this analysis.

\section{DATA ANALYSIS}

Several different variables are employed when studying the source. Fits in one dimension use either $Q_{\text {inv }}=\sqrt{\vec{q}^{2}-q_{0}^{2}}$ or $Q_{r=\tau}=\sqrt{\vec{q}^{2}+q_{0}^{2}}$, which assumes a spherical source decaying with time; the interpretation of this is influenced by Lorentz effects which are different for different particle species [18]. Here $q_{i}=k_{1, i}-k_{2, i}$, and $k_{i}$ are the particle momenta. These problems are alleviated by fitting in two dimensions, $Q_{t}$ perpendicular to the beam, and $Q_{l}$ parallel to the beam. The direction perpendicular to the beam may be further resolved into a direction along the momentum sum of the particles, $Q_{t_{o}}$, and perpendicular to this, $Q_{t_{s}}$. Being parallel to the velocities of the particles, $Q_{t_{o}}$ is sensitive to the lifetime of the source [10]. Data are analysed in the frame in which the momentum sum in the beam direction of both particles is zero. This frame complicates the interpretation of $Q_{l}$; however, it couples the lifetime information solely to $Q_{t_{o}}$ [10]. Data in $Q_{r=\tau}$ are analysed in the nucleon-nucleon centre-of-mass frame to allow comparison with Experiment E802 [18] at the Brookhaven AGS.

Two different spectrometer settings are used, called horizontal and vertical, which optimize the acceptance in $Q_{t_{o}}$ and $Q_{t_{s}}$ respectively. In the horizontal setting for $\mathrm{S}+\mathrm{Pb}$ collisions at $200 \mathrm{GeV} / \mathrm{c}$ per nucleon the data samples contain 30,000 reconstructed $K^{+}$ pairs, 5000 reconstructed $K^{-}$pairs, 43,000 reconstructed $p K^{+}$pairs, and $900 \bar{p} K^{-}$pairs. For $\mathrm{p}+\mathrm{Pb}$ collisions at $450 \mathrm{GeV} / \mathrm{c}$ per nucleon we have $7000 K^{+}$pairs and $4500 \mathrm{p} K^{+}$ pairs. In the vertical setting we have $30,000 K^{+}$pairs for $\mathrm{S}+\mathrm{Pb}$ collisions at $200 \mathrm{GeV} / \mathrm{c}$ per nucleon.

The single-particle acceptance curves for both the horizontal and vertical settings are shown in Fig. 2, and span the rapidity range from 2.9 to 3.3 for the horizontal setting and 2.7 to 3.1 for the vertical setting. The $p_{T}$ range is from 0.0 to $0.7 \mathrm{GeV} / c$. The $K^{+}$pairs from $\mathrm{S}+\mathrm{Pb}$ collisions come from a $0.5 \mathrm{~cm}$ thick target and the rest of the data presented in this paper come from a $1 \mathrm{~cm}$ target. The spectrometer two-particle trigger biases the data towards the $10 \%$ most central collisions as determined by the silicon multiplicity detector.

Tracks are reconstructed from hit positions on the three hodoscopes, with pattern recognition constrained by straight-line trajectories after the magnets. Pions, electrons, and most muons are vetoed based on the Cherenkov pulse heights. The UCAL electromagnetic-to-total energy ratios further veto electrons [19] produced after the Cherenkovs. Figure 2 shows the distribution of the mass-squared calculated from measured momentum and time of flight in $\mathrm{S}+\mathrm{Pb}$ collisions. Well-separated peaks are visible for kaons and protons from $\mathrm{S}+\mathrm{Pb}$ collisions. Contamination of $\mathrm{K}$ pairs by $\mathrm{pK}$ is less than $1 \%$. Contamination of $\mathrm{K}$ pairs by tracks not originating at the target is less than $3 \%$. For the $0.5 \mathrm{~cm}$ target in the horizontal setting the resolution for two particles in $Q_{\text {inv }}$, $Q_{t}, Q_{t_{s}}$, and $Q_{t_{o}}$ is about $20 \mathrm{MeV} / c$ and in $Q_{l}$ it is $10 \mathrm{MeV} / c$. However, we have limited acceptance in $Q_{t_{s}}$ in the horizontal setting and use the vertical setting to measure it with a resolution of $30 \mathrm{MeV} / \mathrm{c}$. These values increase by a few $\mathrm{MeV} / \mathrm{c}$ for data from the $1 \mathrm{~cm}$ target. Bins of size $15 \mathrm{MeV} / \mathrm{c}$ are employed in the one- and two-dimensional fits. In the three-dimensional fits, $20 \mathrm{MeV} / \mathrm{c}$ bins are used to decrease statistical errors. This bin size has been shown, via a Monte Carlo study, to cause negligible errors on the fitted radius parameter of a Gaussian distribution with radius $3 \mathrm{fm}$. 
The correlation function is determined using

$$
C(\vec{q})=\frac{A(\vec{q})}{B(\vec{q})}
$$

The 'real distribution' $A(\vec{q})$ is the distribution of the relative momentum. The background distribution, $B(\vec{q})$, is generated as follows: for each event in $A(\vec{q})$, twenty pairs of events from $A(\vec{q})$ are selected randomly to form the background pairs. In these pairs, one particle in each event is selected randomly to create a new 'event' for the $B(\vec{q})$ distribution. Consequently, the statistical error is dominated by the data sample. As in the real distribution, events from $B(\vec{q})$ are rejected if the tracks pass through the same hodoscope slat. Tracks entering neighbouring slats in $\mathrm{H} 1$ are rejected as well, since the geometry of H1 makes many single particles hit two slats. Thus effects from the loss of close-by pairs cancel for the two-particle correlation. Our procedure ensures that the 'real' and 'background' distributions are from the same class of events.

The background spectrum is distorted compared to the ideal uncorrelated twoparticle spectrum owing to the effect of the two-particle correlations on the single-particle spectrum and is iteratively corrected in the data $[15,20,21]$. The data are further corrected for the distortion of the two-particle spectrum by the momentum resolution of the spectrometer and the two-particle acceptance of the detectors. These corrections depend on the source size and the fitting results, so an iterative approach with a Gaussian source distribution is used. The results for these data samples converge inside the experimental statistical error within four iterations. Furthermore, two-particle correlations arising from Coulomb interactions are corrected by the standard Gamow correction. Corrections based on Coulomb wave-function integration [22] increase the extracted radius parameter by less than $10 \%$ but do not significantly affect the lambda parameter defined in Eq. (2). Coulomb interactions with the residual nuclear system are neglected but should be small for two particles with identical charge-to-mass ratios which experience similar accelerations in the Coulomb field. Final-state strong interactions are also expected to be small if the emitted particles have similar charge-to-mass ratios, but owing to large uncertainties in proposed procedures, no corrections are applied for them [23].

The data are fit with:

$$
\begin{gathered}
C\left(Q_{\text {inv }}\right)=D\left[1+\lambda \exp \left(-Q_{i n v}^{2} R_{i n v}^{2}\right)\right] \\
C\left(Q_{r=\tau}\right)=D\left[1+\lambda \exp \left(-\left(\vec{q}^{2}+q_{0}^{2}\right) R_{r=\tau}^{2}\right)\right] \\
C\left(Q_{t}, Q_{l}\right)=D\left[1+\lambda \exp \left(-Q_{t}^{2} R_{t}^{2}-Q_{l}^{2} R_{l}^{2}\right)\right] \\
C\left(Q_{t_{o}}, Q_{t_{s}}, Q_{l}\right)=D\left[1+\lambda \exp \left(-Q_{t_{o}}^{2} R_{t_{o}}^{2}-Q_{t_{s}}^{2} R_{t_{s}}^{2}-Q_{l}^{2} R_{l}^{2}\right)\right]
\end{gathered}
$$

for the Gaussian parametrization and

$$
C\left(Q_{i n v}\right)=D\left[1+\lambda \exp \left(-2 Q_{i n v} R_{i n v}\right)\right]
$$

for the exponential parameterization. We use the least-squares method to find the values of the unknown parameters

$$
\chi^{2}=\sum_{i, j}\left(C_{i}-A_{i} / B_{i}\right) V_{i j}^{-1}\left(C_{j}-A_{j} / B_{j}\right)
$$

where $V_{i j}$ is the covariance matrix defined below and the summing is done over bins $(i, j)$ where there are more than 10 counts in the 'reals' $\left(A_{i}\right)$ distribution. This affects bins at 
Table 1: Fitted results of Gaussian parametrizations in $Q_{\text {inv }}$

\begin{tabular}{|c|c|c|c|c|}
\hline System & $\lambda$ & $R_{\text {inv }}(\mathrm{fm})$ & $\chi^{2} / N_{\text {dof }}$ & Setting \\
\hline $\mathrm{SPb} K^{+} K^{+}$ & $0.79 \pm 0.05$ & $2.97 \pm 0.12$ & $99 / 69$ & Horizontal + vertical \\
\hline $\mathrm{SPb} K^{+} K^{+}$ & $0.92 \pm 0.08$ & $3.22 \pm 0.20$ & $53 / 31$ & Horizontal \\
\hline $\mathrm{SPb} K^{-} K^{-}$ & $1.06 \pm 0.15$ & $3.06 \pm 0.29$ & $30 / 28$ & Horizontal \\
\hline $\mathrm{pPb} K^{+} K^{+}$ & $0.68 \pm 0.06$ & $1.71 \pm 0.17$ & $65 / 54$ & Horizontal + Vertical \\
\hline
\end{tabular}

Table 2: Fitted results of exponential parametrizations in $Q_{\text {inv }}$

\begin{tabular}{|c|c|c|c|c|}
\hline System & $\lambda$ & $R_{\text {inv }}(\mathrm{fm})$ & $\chi^{2} / N_{\text {dof }}$ & Setting \\
\hline $\mathrm{SPb} K^{+} K^{+}$ & $1.50 \pm 0.10$ & $2.47 \pm 0.14$ & $91 / 69$ & Horizontal + Vertical \\
\hline $\mathrm{SPb} K^{+} K^{+}$ & $1.80 \pm 0.18$ & $2.64 \pm 0.22$ & $26 / 31$ & Horizontal \\
\hline $\mathrm{SPb} K^{-} K^{-}$ & $2.11 \pm 0.36$ & $2.68 \pm 0.37$ & $29 / 28$ & Horizontal \\
\hline $\mathrm{pPb} K^{+} K^{+}$ & $1.10 \pm 0.10$ & $1.04 \pm 0.19$ & $55 / 54$ & Horizontal + Vertical \\
\hline
\end{tabular}

high $Q$ at the edge of our acceptance. Maximum likelihood estimators reduce to leastsquares estimators in this limit. Fitting the three-dimensional equation requires using data from two different spectrometer settings, horizontal and vertical, and the data sets are fit simultaneously. To allow comparison with the $\mathrm{S}+\mathrm{Pb} \rightarrow K^{-} K^{-}$and $\mathrm{p}+\mathrm{Pb} \rightarrow K^{+} K^{+}$ horizontal setting data, the $\mathrm{S}+\mathrm{Pb} \rightarrow K^{+} K^{+}$data are also fit solely with horizontal setting data.

Systematic errors are evaluated by varying analysis parameters and seeing the difference in the correlation functions produced. First, the cut of neighbouring-slat hits on $\mathrm{H} 1$ is extended to $\mathrm{H} 2$ and $\mathrm{H} 3$ and the two-particle acceptance correction recalculated. Second, the assumed momentum resolution is increased by $20 \%$ when correcting the data. The error matrix is calculated from

$$
V_{i j}^{s y s}=\left[\frac{\sum_{k=1}^{N} C_{i k} C_{j k}}{N}-C_{i}^{\text {mean }} C_{j}^{\text {mean }}\right] \frac{N}{N-1},
$$

where $N$ is equal to the number of simulated data sets and

$$
V_{i j}=V_{i j}^{s y s}+V_{i j}^{\text {stat }}, \quad V_{i j}^{\text {stat }}=0 \text { if } i \neq j
$$

For our data the correlation coefficient $\rho$ :

$$
\rho_{i j}=V_{i j} /\left(V_{i i} V_{j, j}\right)^{\frac{1}{2}}
$$

is less than 0.2 for almost all bins, indicating that the systematic errors have little effect on fits to the data.

No correction is made for the $1 \%$ contamination of the data sets by Kp events or K $\mu$ events.

\section{RESULTS}

Fits to $Q_{\text {inv }}$ are performed on all the data sets; the results for the horizontal spectrometer setting are summarized in Table 1 and shown together with the data in Fig. 3. Although fits to Gaussians and exponentials in $Q_{i n v}$ yield similar chi-squares (see tables 1-2), it is possible that the correlation is neither Gaussain nor exponential. In Fig. 4 one can see how the correlation changes with the corrections applied. Low $Q_{\text {inv }}$ bins are 
Table 3: Fitted results of Gaussian parametrizations in $Q_{r=\tau}$

\begin{tabular}{|c|c|c|c|c|c|}
\hline System & $\lambda$ & $R(\mathrm{fm})$ & $\chi^{2} / N_{\text {dof }}$ & Setting & $\left\langle p_{T}\right\rangle(\mathrm{MeV} / c)$ \\
\hline $\mathrm{SPb} K^{+} K^{+}$ & $0.78 \pm 0.04$ & $2.62 \pm 0.10$ & $99 / 76$ & Hor. + ver. & 246 \\
\hline $\mathrm{SPb} K^{+} K^{+}$ & $0.85 \pm 0.06$ & $2.69 \pm 0.14$ & $65 / 36$ & Horizontal & 230 \\
\hline $\mathrm{SPb} K^{-} K^{-}$ & $0.99 \pm 0.13$ & $2.70 \pm 0.27$ & $27 / 32$ & Horizontal & 230 \\
\hline $\mathrm{pPb} K^{+} K^{+}$ & $0.60 \pm 0.06$ & $1.45 \pm 0.14$ & $59 / 68$ & Hor. + ver. & 237 \\
\hline $\mathrm{SPb} K^{+} K^{+}$ & $0.90 \pm 0.06$ & $2.63 \pm 0.13$ & $66 / 36$ & Horizontal & $243(\geq 50)$ \\
\hline
\end{tabular}

Table 4: Fitted results of Gaussian parametrizations in $Q_{t}$ and $Q_{l}$

\begin{tabular}{|c|c|c|c|c|c|}
\hline System & $\lambda$ & $R_{t}(\mathrm{fm})$ & $R_{l}(\mathrm{fm})$ & $\chi^{2} / N_{\text {dof }}$ & Setting \\
\hline $\mathrm{SPb} K^{+} K^{+}$ & $0.84 \pm 0.04$ & $2.78 \pm 0.11$ & $3.24 \pm 0.21$ & $526 / 466$ & Horizontal + vertical \\
\hline $\mathrm{SPb} K^{+} K^{+}$ & $0.94 \pm 0.06$ & $2.83 \pm 0.16$ & $3.32 \pm 0.22$ & $270 / 255$ & Horizontal \\
\hline $\mathrm{SPb} K^{-} K^{-}$ & $1.18 \pm 0.18$ & $3.24 \pm 0.39$ & $3.66 \pm 0.62$ & $78 / 94$ & Horizontal \\
\hline
\end{tabular}

overcorrected by the Gamow correction in the second picture as it has no correction for our finite momentum resolution, which shifts data from higher to lower $Q_{\text {inv }}$. The $R_{\text {inv }}$ parameter increases from $\mathrm{p}+\mathrm{Pb}$ collisions to $\mathrm{S}+\mathrm{Pb}$ collisions and is smaller than for pions in similar systems $[15,24]$. For $\mathrm{S}+\mathrm{Pb}$ collisions the $K^{+} K^{+}$and $K^{-} K^{-} R_{\text {inv }}$ parameters are equal within errors. The $\lambda$ parameter is also larger than that for pions. These trends are supported by source parameters extracted from $Q_{r=\tau}$ and two-dimensional fits in $Q_{t}$ and $Q_{l}$ seen in Tables 3 and 4. Projections of the fits into $Q_{t}$ and $Q_{l}$ are presented in Fig. 5. The acceptance of the horizontal setting is such that $R_{t}$ is close to $R_{t_{o}}$.

Three-dimensional fits are performed on the $\mathrm{S}+\mathrm{Pb} \rightarrow K^{+} K^{+}$data set and results are shown in Table 5. Projections onto the three axes are presented in Fig. 6 . The $\mathrm{S}+\mathrm{Pb} \rightarrow K^{-} K^{-}$and the $\mathrm{p}+\mathrm{Pb} \rightarrow K^{+} K^{+}$data sets are limited solely to the horizontal setting thus prohibiting three-dimensional fits. Note that $R_{t_{o}}$ is similar to $R_{t_{s}}$. The radius $R_{t_{o}}$ measures $\approx \sqrt{R_{T}^{2}+\beta^{2} \tau^{2}}$ where $R_{T}$ is the transverse radius of a cylindrical source $\left(\approx R_{t_{s}}\right), \beta$ is the average velocity of the pair, and $\tau$ is the lifetime of the source. For $\mathrm{S}$ $+\mathrm{Pb} \rightarrow \pi^{+} \pi^{+}$collisions $R_{t s}$ is found to be $4.14 \pm 0.27 \mathrm{fm}$ [25] which is larger than for kaons, and $\lambda$ is found to be $0.56 \pm 0.02$ which is smaller than for kaons.

To investigate the effect of the $1 \%$ contamination of the KK data set by Kp, a correlation is constructed from Kp pairs assuming both are kaons. As expected, the correlations are flat as seen in Fig. 7 .

\section{DISCUSSION}

The results on correlations of $\mathrm{K}^{+}$and $\mathrm{K}^{-}$pairs have long been awaited for two reasons: the relative freedom from resonance decay products and the opportunity to compare the $\mathrm{K}^{+}$which has a small nucleon-scattering cross-section with the $\mathrm{K}^{-}$which has

Table 5: Fitted results of Gaussian parametrizations in $Q_{t_{o}}, Q_{t_{s}}$, and $Q_{l}$, using the horizontal and vertical settings.

\begin{tabular}{|c|c|c|c|c|c|c|}
\hline System & $\lambda$ & $R_{t_{o}}(\mathrm{fm})$ & $R_{t_{s}}(\mathrm{fm})$ & $R_{l}(\mathrm{fm})$ & $\chi^{2} / N_{\text {dof }}$ & $\left\langle p_{T}\right\rangle(\mathrm{MeV} / c)$ \\
\hline $\mathrm{SPb} K^{+} K^{+}$ & $0.82 \pm 0.04$ & $2.77 \pm 0.12$ & $2.55 \pm 0.20$ & $3.02 \pm 0.20$ & $925 / 1011$ & 246 \\
\hline $\mathrm{SPb} K^{+} K^{+}$ & $0.83 \pm 0.06$ & $3.05 \pm 0.23$ & $2.44 \pm 0.44$ & $3.44 \pm 0.29$ & $258 / 293$ & 163 \\
\hline $\mathrm{pPb} K^{+} K^{+}$ & $0.70 \pm 0.07$ & $1.53 \pm 0.17$ & $1.22 \pm 0.76$ & $2.40 \pm 0.30$ & $119 / 121$ & 237 \\
\hline
\end{tabular}


a considerably larger cross-section. Recently, the microscopic physics simulations which include rescattering (RQMD [13] and ARC [26]) have been shown to reproduce the inclusive particle spectra in $p_{T}$ and rapidity. The strong correlation between position and momentum which is manifested in these calculations may be interpreted as a collective expansion, which would profoundly alter the interpretation of the correlations. If the simulations reproduce the experimental correlation functions, we may use them to estimate the physical source size at freeze-out via the particle positions in the simulations themselves. Typically, we find that the radius parameters are smaller than the source size at freeze-out, and $p_{T}$ dependent. We discuss these three items in turn.

The correlation functions we measure in $\mathrm{S}+\mathrm{Pb}$ collisions for $\mathrm{K}^{+}$and $\mathrm{K}^{-}$are similar, indicating that the different interaction cross-section with nucleons are not important in the central region at SPS energies. This is confirmed by our measurements of the ratio $\mathrm{p} / \pi^{+}=0.12$ [27]. $\mathrm{K} \pi$ scattering dominates, which is similar for $\mathrm{K}^{+}$and $\mathrm{K}^{-}$. In RQMD simulations $[13] \mathrm{K}^{+}$and $\mathrm{K}^{-}$interact on average the same number of times before freezeout. The simulated correlations are quite similar, in agreement with our data. We conclude that the central region of the $\mathrm{S}+\mathrm{Pb}$ collisions is almost nucleon-free.

We note that the ratio of $\mathrm{K} \pi$ to $\pi \pi$ cross sections is temperature-dependent [28]. However, in the range of temperatures likely to be encountered in our experiment they are quite similar [28]. Consequently, we might expect similar freeze-out radii, but if resonance contributions are greater for pions, the resonance lifetimes will extend the measured radius parameters. The $R_{t s}$ parameter extracted from kaons is smaller than the value of $4.60 \pm$ $0.29 \mathrm{fm}$ from pions [25]; this is also true for samples of pions and kaons selected to have the same $p_{T}$, as shown in Table 5 . The E802/E859 Collaboration measured $\mathrm{K}^{+}$correlations in 14.6 $\mathrm{A} \mathrm{GeV} / c \mathrm{Si}+\mathrm{Au}$ and concluded 'after accounting for the different transformation properties of the pion and kaon pairs, the pion source size remains larger than that for the kaons, although statistical and systematic uncertainties are comparable to the difference.'[18]

Another effect of the resonances is to decrease the magnitude of the correlations at low $\mathrm{Q}$, giving us a further handle on the resonance contributions via the parameter $\lambda$. RQMD predicts that $\approx 27 \%$ of pions, $\approx 5 \%$ of $\mathrm{K}^{{ }^{\prime}}{ }_{\mathrm{s}}$ and $\approx 3 \%$ of $\mathrm{K}^{{ }^{\prime}}{ }_{\mathrm{s}}$ near mid-rapidity $(2<y<4)$ come from the decay of resonances with lifetimes $c \tau>20 \mathrm{fm}$. These resonance contributions increase by a factor of $\approx 1.5$ for pions and kaons in the NA44 acceptance [14]. We find $\lambda_{\pi}$ about $2 / 3$ of $\lambda_{K}$ [25], indicating a smaller resonance effect with kaons, in approximate agreement with the results from RQMD.

The two topics discussed above do not depend on a model of the collision dynamics, which becomes important for the interpretation of the 'radius' parameters obtained in the fits. The sensitivity of the identical-particle correlations to a dynamical correlation between position and momentum has long been known from the example of the effect of the inside-outside cascade in the longitudinal direction in hadron-hadron collisions, where the measured radius parameter is much smaller than the actual physical extension of the position of the particles formed by hadronization. Collective motion has a different origin, but leads to the same kinematical result. Since radial expansion would cause correlations in the transverse direction as well, care is needed to relate the measured 'radius' parameters in our fits to the physical source sizes. Accordingly, we refer to them as 'R-parameters' and discuss what they imply about the source sizes.

As a first step, we compare the pattern of the R-parameters from $\mathrm{p}+\mathrm{Pb}$ and $\mathrm{S}$ $+\mathrm{Pb}$ collisions in Table 5 . In $\mathrm{p}+\mathrm{Pb}$ it is natural to expect in the central region little rescattering, thermalization, or collective motion, so the transverse motion should be very 
different from that along the beam. We see that the $R_{l}$ is quite different from $R_{t 0}$ and $R_{t s}$. In $\mathrm{S}+\mathrm{Pb}$ collisions the three $\mathrm{R}$-parameters are nearly equal, suggesting significant influence from thermalization and collective motion. The simplest way to interpret the $\mathrm{R}$-parameters in terms of the source size would be to compare the size of the projectile nucleus to the measured $R_{t s}$. Since the nucleus is well described as a hard sphere of constant density, one should fit the correlation data with a hard sphere form rather than a Gaussian. This involves multiplying our results by $\sqrt{5}$. One then finds an experimental $R_{t s}($ hard sphere $)=5.7 \pm 0.4 \mathrm{fm}$ for kaons compared to $\mathrm{r}($ sulphur $)=3.8 \mathrm{fm}$. Since the position-momentum correlation will decrease the extracted $R_{t s}$ compared to the true transverse source size, we would then conclude that the source in the final state is substantially larger than the projectile. It is less clear which projectile size to choose for a $p+$ $\mathrm{Pb}$ collision, but if we were to use $\mathrm{r}$ (proton) $=1 \mathrm{fm}$, we would reach a similar conclusion. Microscopic simulations show that the distribution of particle positions at freeze-out is more Gaussian than hard sphere. This complicates the above argument, and leads us to study an interpretation consistent with the characteristics of the simulation.

We compare the experimental results with the RQMD event generator [13] and filter simulating the acceptance of NA44. Figure 8 compares the shape of the $K^{+} K^{+}$correlation function from the generator and data; the RQMD events are selected on event multiplicity to match the NA44 trigger. The upper left shows the correlation function in $Q_{\text {inv }}$, and the other three panels in $Q_{l}, Q_{t o}$, and $Q_{t s}$. The agreement between RQMD and data is fair, though RQMD tends to predict somewhat smaller R-parameters (wider correlation function). We can fit the RQMD correlation function with a Gaussian-distributed source to simplify the comparison, even though the shape of the predicted correlation function is complicated, in part due to resonance decays. We perform this comparison using $R_{t s}$, as this most closely reflects the true source size in the transverse direction [11]. $R_{t s}$ from $\mathrm{K}^{+} \mathrm{K}^{+}$is $2.38 \pm 0.15 \mathrm{fm}$ for $\mathrm{S}+\mathrm{Pb}$ RQMD events. We can then use RQMD to relate the measured $R_{t s}$ parameter to the actual transverse source size that we wish to determine by using the width $(\sigma)$ of a Gaussian fit to the transverse position distribution of $\mathrm{K}^{+}$ at freeze-out in the model, which is $2.9 \mathrm{fm}$. We see that the true transverse size is only about $20 \%$ larger than $R_{t s}$ for $\mathrm{K}^{+} \mathrm{K}^{+}$correlations. The source size is indeed larger than the projectile. For $\pi \pi$ correlations, the effect of the collective motion is larger, and the $p_{T}$ dependence of the R-parameters can provide additional information [29].

Using RQMD we can investigate how resonances can change the extracted radius parameters from correlations by turning off all resonances in the code. We find that the kaon $R_{t s}$ parameter decreases by about $10 \%$ and the pion radius by about $20 \%$. This does not fully account for the difference between the radius parameters for kaons and pions measured at the same transverse momentum.

\section{CONCLUSIONS}

We have investigated $K^{+}$and $K^{-}$correlation functions at mid-rapidity and found them to be similar, indicating a nucleon-free central region in our collisions. Extracted radius parameters for kaons are smaller than those for pions. Resonance decays to pions can in part account for this difference. The transverse position distribution of kaons at freeze-out given by RQMD shows that the extracted radius parameters set a lower limit on the true source size. The radii we measure for $\mathrm{S}+\mathrm{Pb}$ and $\mathrm{p}+\mathrm{Pb}$ collisions are both larger than the projectile, signalling the presence of collective expansion. Correlation functions from RQMD, which has significant rescattering among the particles produced before freeze-out, agree reasonably well with our data. 


\section{Acknowledgements}

The NA44 Collaboration wishes to thank the staff of the CERN PS-SPS accelerator complex for their excellent work. We thank the technical staff at CERN and the collaborating institutes for their valuable contributions. We are also grateful for the support given by the Österreichische Fonds zur Förderung der Wissenschaftlichen Forschung (Grant P 6312P); the Science Research Council of Denmark; the Japanese Society for the Promotion of Science, and the Ministry of Education, Science and Culture, Japan; the Science Research Council of Sweden; the US Department of Energy, and the National Science Foundation (Nuclear Physics) through grants PHY8906284 and PHY8958491.

\section{References}

[1] R. Hanbury-Brown and R. Q. Twiss, Nature 178 (1956) 1046.

[2] M. Gyulassy, S. K. Kauffmann and L. W. Wilson, Phys. Rev. C20 (1979) 2267.

[3] B. Lorstad, Int. J. Mod. Phys. A4 (1988) 2861.

[4] M. Gyulassy and S. Padula, Phys. Rev. C41 (1990) 21.

[5] S. Pratt, P.J. Siemens and A. P. Vischer, Phys. Rev. Lett. 68 (1992) 1109.

[6] S. Schnetzer et al., Phys. Rev. Lett. 49 (1982) 989.

[7] S. Pratt, Phys. Rev. D33 (1986) 1314.

[8] G. Bertsch and G. E. Brown, Phys. Rev. C40 (1989) 1830.

[9] B. Andersson and W. Hofmann, Phys. Lett. B169 (1986) 364.

[10] S. Pratt, T. Csörgö and J. Zimányi, Phys. Rev. C42 (1990) 2646.

[11] J. Sullivan et al., Phys. Rev. Lett. 70 (1992) 3000.

[12] R. Lednicky and T.B. Progulova. Z. Phys. C55 (1992) 295.

[13] H. Sorge, H. Stöcker and W. Greiner, Nucl. Phys. A498 (1989) 567.

[14] J. Sullivan et al., Nucl. Phys. A566 (1994) 531c.

[15] H. Beker et al., Phys. Lett. B302 (1993) 510.

[16] N. Maeda et al., to be published.

[17] T. Kobayashi and T. Sugitate, Nucl. Instrum. Methods A287 (1990) 389.

[18] Y. Akiba et al., Phys. Rev. Lett 70 (1993) 1057.

[19] T. Akesson et al., Nucl. Instrum. Methods A262 (1987) 243.

[20] K. Kadija and P. Seyboth, Phys. Lett. B287 (1992) 363.

[21] W. A. Zajc et al., Phys. Rev. C29 (1984) 2173.

[22] S. Pratt, Phys. Rev. D33 (1986) 72.

[23] D. Boal, C. Gelbke and B. Jennings, Rev. Mod. Phys. 62 (1990) 553.

[24] M. Lahanas et al., Nucl. Phys. A525 (1991) 327c.

[25] H. Beker et al., Directional analysis of identified pion interferometry at the CERN SPS, to pe published.

[26] Y. Pang, T. Schlagel, and S. Kahana, Phys. Rev. Lett 68 (1992) 2743.

[27] M. Murray et al., Nucl. Phys. A566 (1994) 515c.

[28] K. Haglin and S. Pratt, Phys. Lett $\mathbf{B}$ in press.

[29] H. Beker et al., $m_{T}$ dependence of boson interferometry in heavy ion collisions at the CERN SPS, to be published. 

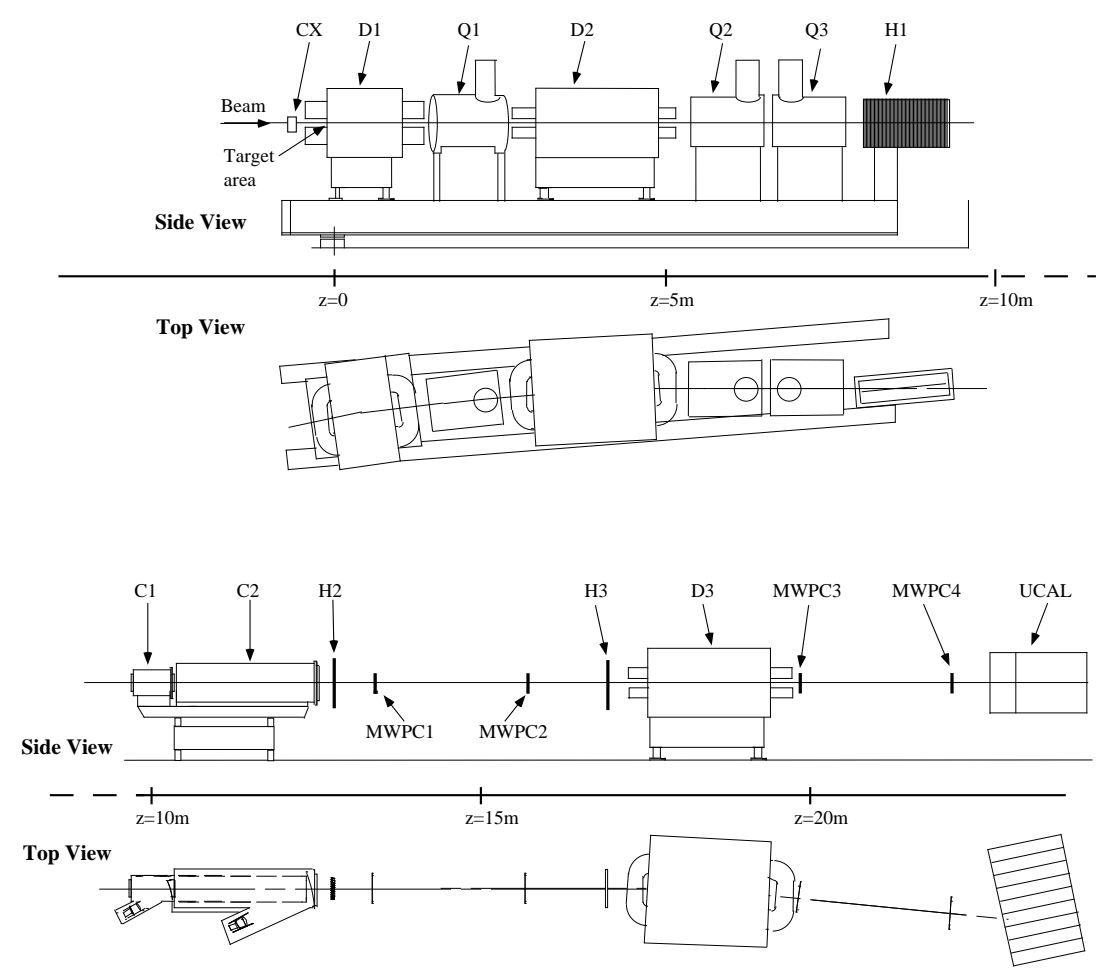

Figure 1: The NA44 experimental set-up.
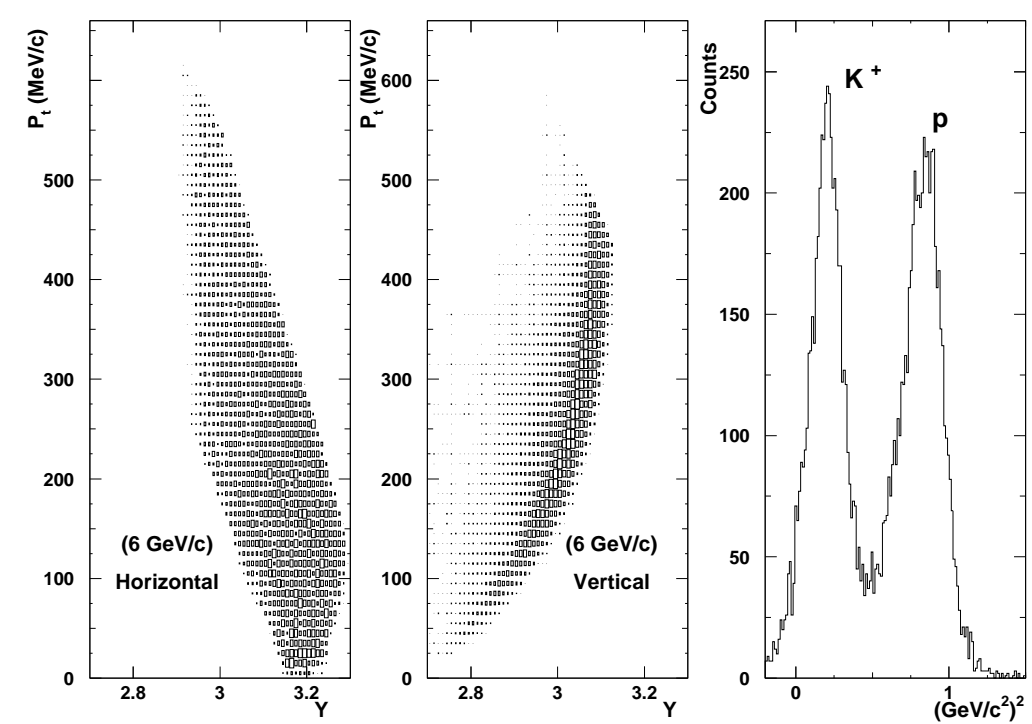

Figure 2: The NA44 acceptance and mass squared plots. 

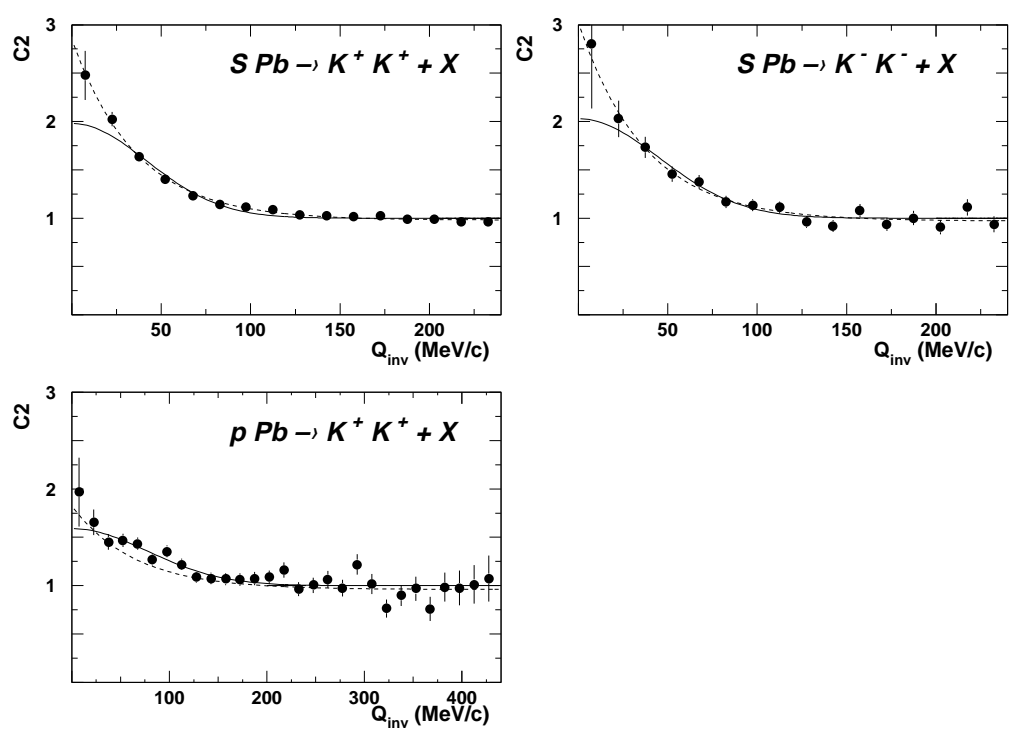

Figure 3: The $Q_{\text {inv }}$ plots from the horizontal setting. The solid line shows the Gaussian fit and the dashed line shows the exponential fit. Error bars are statistical.
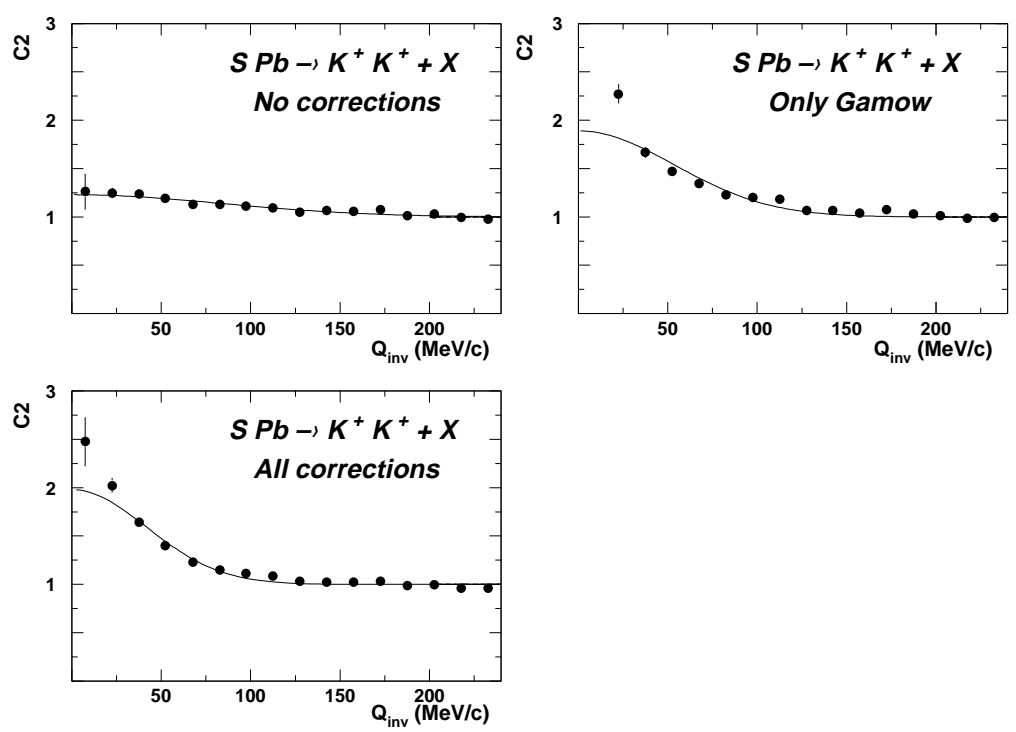

Figure 4: The change in the correlation with corrections for the horizontal setting. The solid line shows the Gaussian fit and error bars are statistical. 

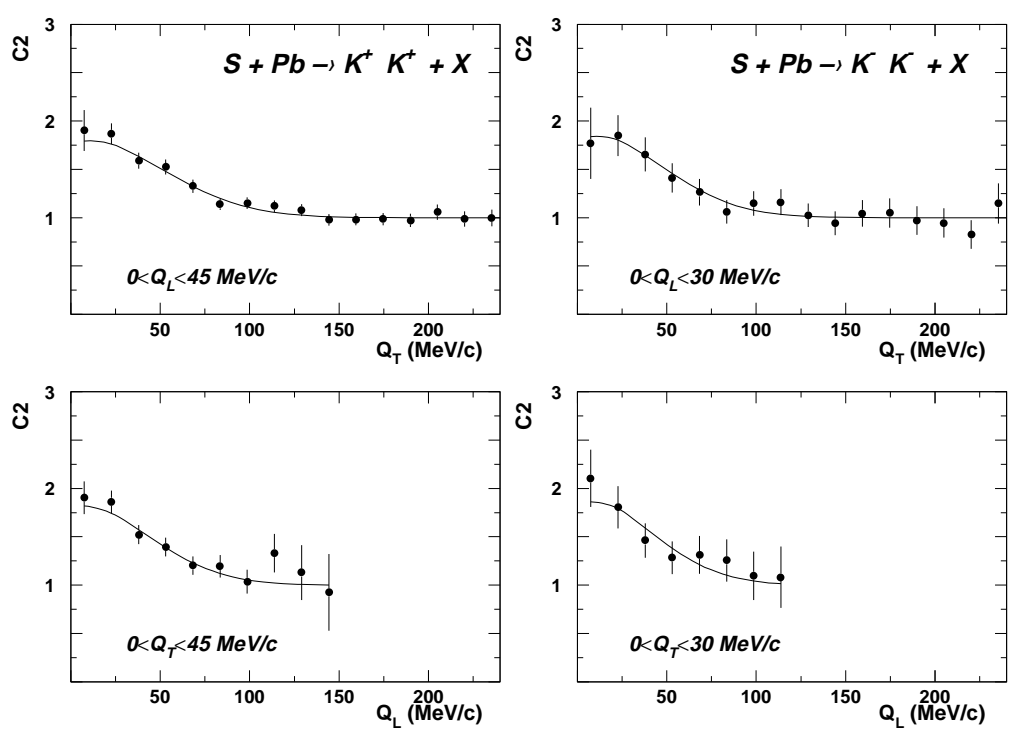

Figure 5: The $Q_{t}$ and $Q_{l}$ projections from the horizontal setting. The lines are from a Gaussian fit. Error bars are statistical.
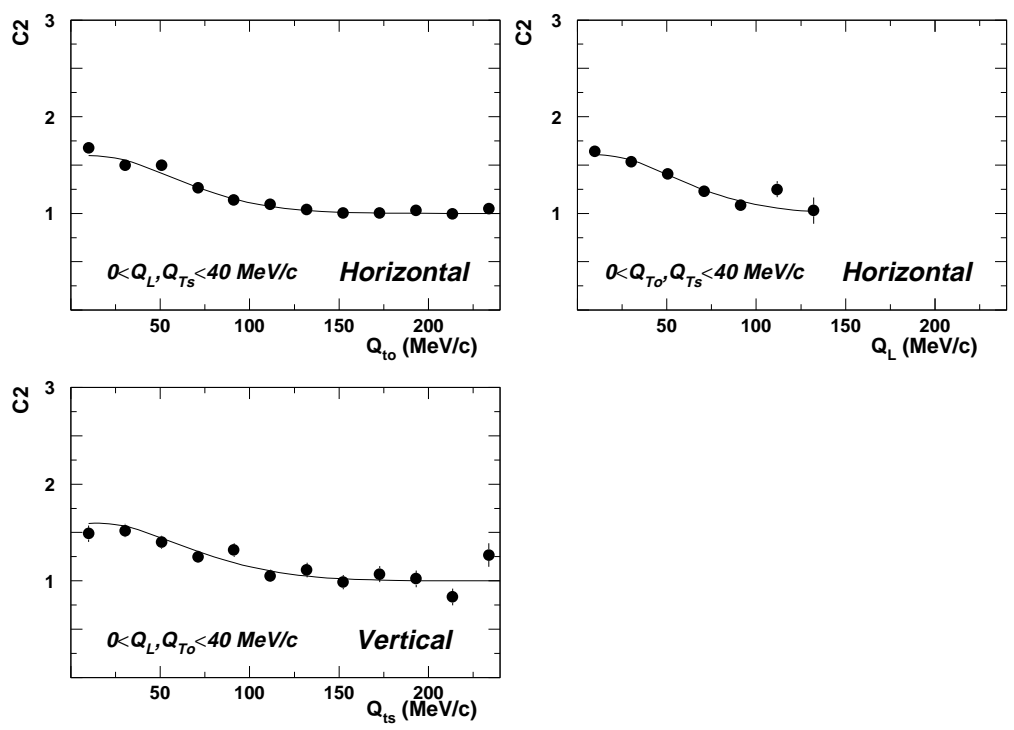

Figure 6: The $Q_{t_{o}}, Q_{t_{s}}$, and $Q_{l}$ projections for $\mathrm{S}+\mathrm{Pb} \rightarrow K^{+} K^{+}+\mathrm{X}$ collisions. The lines are from a Gaussian fit. Error bars are statistical. 

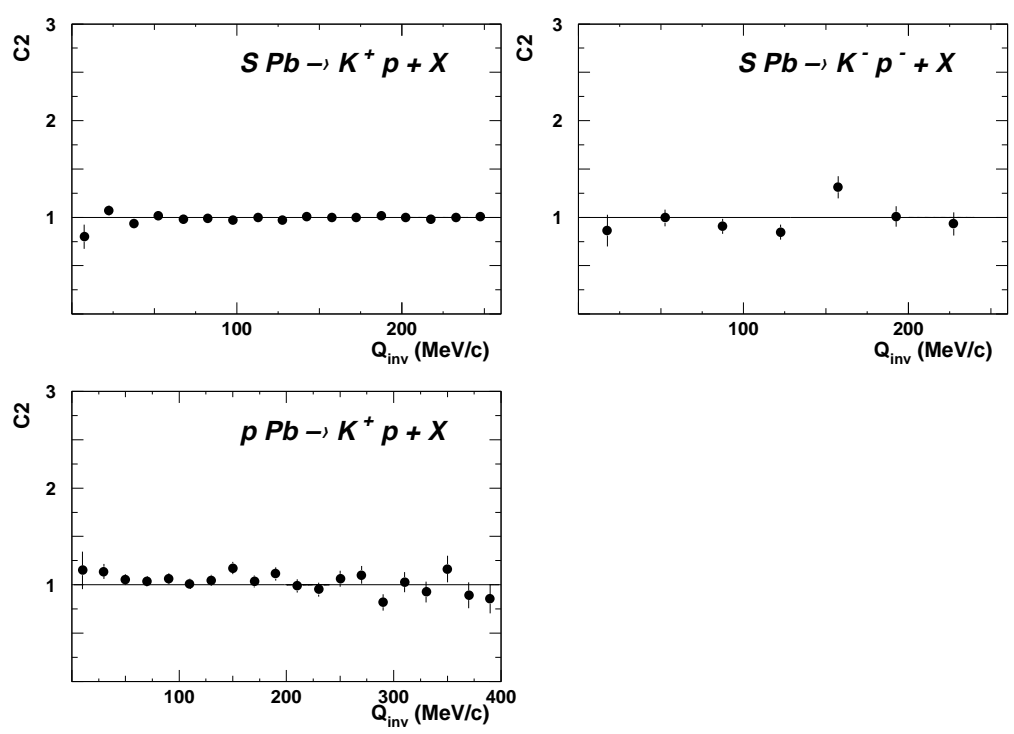

Figure 7: The $\mathrm{p} K^{+}$and $\mathrm{p}^{-} K^{-}$correlation plots from the horizontal setting assuming both are kaons. The lines are to guide the eye. Error bars are statistical.
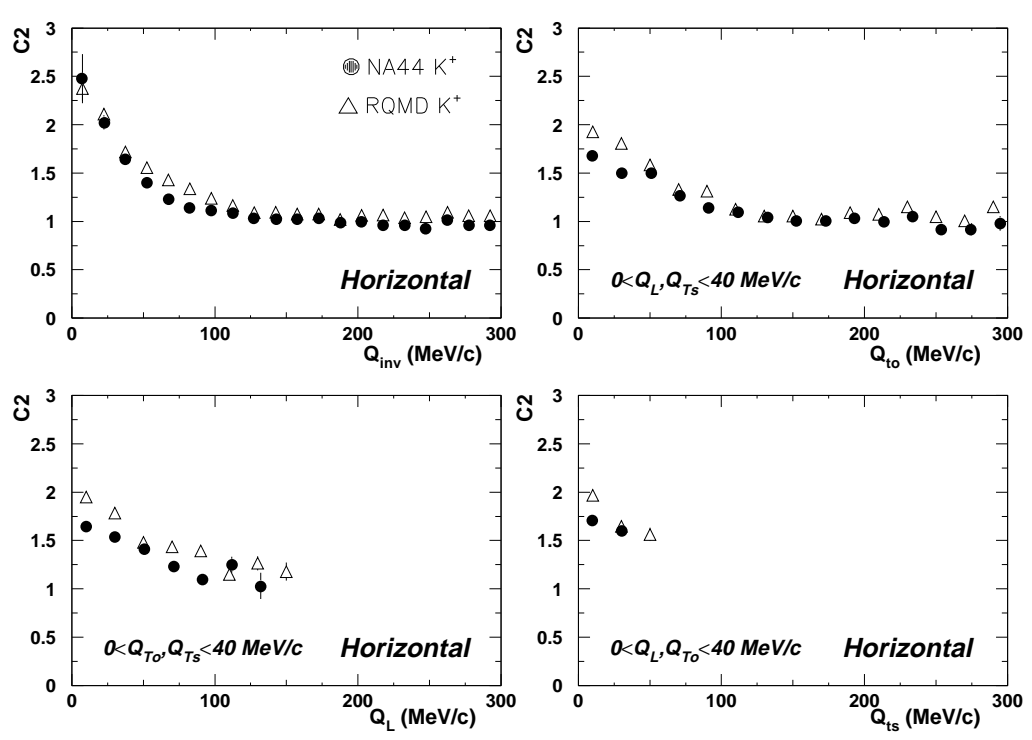

Figure 8: Comparison of correlations generated by RQMD with the data for $\mathrm{S}+\mathrm{Pb} \rightarrow K^{+} K^{+}+\mathrm{X}$ collisions. Error bars are statistical. 